\title{
Four-level Simulation Model of Photovoltaic Matrix
}

\author{
Dawei Liu", Japeng $\mathrm{Su}^{2}$, Zhihao $\mathrm{Li}^{2}$, Anjun Jin ${ }^{1,2, ~ * ~}$ \\ ${ }^{1}$ Solar Thermal Energy Division, Hua-Neng Clean Energy Research Inst, Beijing, China \\ ${ }^{2}$ The Maritime Faculty, Ningbo University, Ningbo, China
}

\author{
Email address: \\ ajjin@nbu.edu.cn (Anjun Jin) \\ ${ }^{*}$ Corresponding author
}

\section{To cite this article:}

Dawei Liu, Japeng Su, Zhihao Li, Anjun Jin. Four-level Simulation Model of Photovoltaic Matrix. American Journal of Science, Engineering and Technology. Special Issue: Advances in Thermoelectric Generation and Renewable Energies. Vol. 5, No. 2, 2020, pp. 89-95.

doi: $10.11648 /$ j.ajset.20200502.16

Received: MM DD, 2020; Accepted: MM DD, 2020; Published: June 18, 2020

\begin{abstract}
Due to the comprehensive influence of many factors such as performance difference of modules and weather environment, the power prediction technology of DC side of photovoltaic (PV) power station has always been a difficult and important research direction. In this paper, a four-level modelling method of photovoltaic matrix is proposed, which can study the influence of the performance deviation of the PV modules on the output power of the array at any position. Compared with the previous research, this work modelling an independent MPPT unit and numbering each battery in series and parallel components, so as to study the influence of component performance deviation on the overall output power in different circuit positions. At the same time, the function of electrical protection inside and outside the components is considered, which makes the calculation results closer to the actual situation of the power plant. By using this method, the influence of different installation direction of modules on the overall power generation of a $50 \mathrm{KW}$ photovoltaic matrix under the same occlusion condition is studied. The results show that when the light intensity loss in the occluded area is less than $40 \%$, there is little difference between the two installation methods; with the increase of the light intensity loss, the power loss of the transverse installation method is smaller.
\end{abstract}

Keywords: Photovoltaic Power Station, Power Generation Prediction, Theoretical Model, Simulation Calculation, Light Shielding

\section{Introduction}

Photovoltaic power prediction technology is very important for the safe and efficient grid connected operation of photovoltaic power station. Among them, the inverter grid side (AC side) can be relatively easy to achieve accurate prediction according to the data provided by the equipment manufacturer, while the power of the component bus side (DC side) has been widely concerned in recent years due to many factors affecting the prediction technology [1]. Although the module manufacturers will provide the photoelectric data of photovoltaic modules, there may be some differences in the actual power generation performance between modules due to different batches, aging and use environment [2]. In addition to the factory performance deviation of photovoltaic modules, the specific reasons for the difference may include: damage to the battery, temperature change, local shadow, cracking of the package layer of modules and discoloration of EVA materials, etc. [3]. Most of the impacts caused by these reasons will gradually become significant with the increase of the power plant scale.

At present, the simulation calculation of the power generation of photovoltaic modules is basically based on the equivalent circuit model of photovoltaic cells [4-6]. According to the model, we can get the relationship between the voltage and current of the single photovoltaic cell in different working conditions, and then through circuit calculation, we can get the output characteristics of photovoltaic modules or arrays. References [7] and [8] provide a method to approximate the explicit expression of the equivalent circuit. By using some special points in the IV curve, the physical parameters that are inconvenient to be measured in the model can be transformed into the electrical parameters that are convenient to be measured, which greatly 
reduces the difficulty of modelling and the amount of calculation. References [9] expand the equivalent circuit model, and consider the reverse breakdown curve of the battery, so that the model In reference $[10,11]$, the output characteristics of the series parallel hybrid circuit of photovoltaic modules are studied by using this model, and the influence of different light blocking modes on the simulation output power is compared, which is close to the actual operation of the power plant.

On the basis of previous work, this paper establishes a four level structure model from cell to square array, which is used to calculate the power of the module side of photovoltaic power station. Compared with the previous research, its main characteristics are as follows: (1) modelling an independent MPPT unit and numbering each battery in series and parallel components, so as to study the influence of component performance deviation on the overall output power in different circuit positions; (2) according to the hierarchical structure of battery component group series array. The structure sequence is modelled, and the electrical protection measures (bypass protection, current anti reaction, etc.) at the level of connection are considered, which makes the calculation results closer to the actual situation of the power plant. Using this modelling method, we also studied the influence of different installation directions of components on the overall power generation of a $50 \mathrm{KW}$ photovoltaic matrix under the same shielding conditions.

\section{Four Level Model of Photovoltaic Power Plant}

\subsection{Hierarchical Structure of Photovoltaic Power Plant}

The photovoltaic matrix that can independently carry out MPPT is the basic unit of generating power prediction on the module side of photovoltaic power station, generally including four hierarchies of battery module string array. The batteries are connected in series to form components. At the same time, each battery has a bypass diode to protect the hot spot effect; the components are connected in series to form a string; the strings are connected in parallel to form a square array, and at the same time, the current counter protection is carried out in the combiner box; finally, the square array is integrated into the inverter for the overall MPPT. The following describes the modelling methods of each level and between levels.

Figure 1 shows the characteristic IV curve of crystalline silicon photovoltaic cells after expanding the range of voltage and current, which occupies the first, second and fourth quadrants: (1) the range of the first quadrant includes the range of voltage from 0 to open circuit voltage $\left(U_{O C}\right)$, current from short circuit current $\left(I_{S C}\right)$ to 0 , that is, the "photovoltaic IV curve" in a general sense. (2) When the voltage continues to rise above $U_{O C}$, the current begins to turn negative and rapidly decreases, entering the fourth quadrant [9]. When several groups of batteries are connected in series and parallel, when the open circuit voltage of one group or a few groups of batteries is significantly lower than that of other groups of batteries, the IV curve of batteries in these low-voltage groups may enter the fourth quadrant. At this time, the current direction of this string is opposite to other strings, and each battery is in the charged power supply. This phenomenon can be avoided if the series parallel connection passes through the combiner box with anti-reverse diode. (3) When the voltage drops to a negative value, the IV curve will enter the second quadrant. At first, the current almost remains the same, but if the voltage drops below a critical value (i.e. avalanche voltage of PN junction of photovoltaic cell, $U_{a}$ ), the current will increase rapidly and the photovoltaic cell will be reverse broken down. When several batteries are connected in series, if the short-circuit current of one or a few batteries is significantly lower than that of other batteries, the IV curve of these ISC smaller batteries may enter the second quadrant. This can be avoided if there is a bypass diode on the series circuit between batteries.

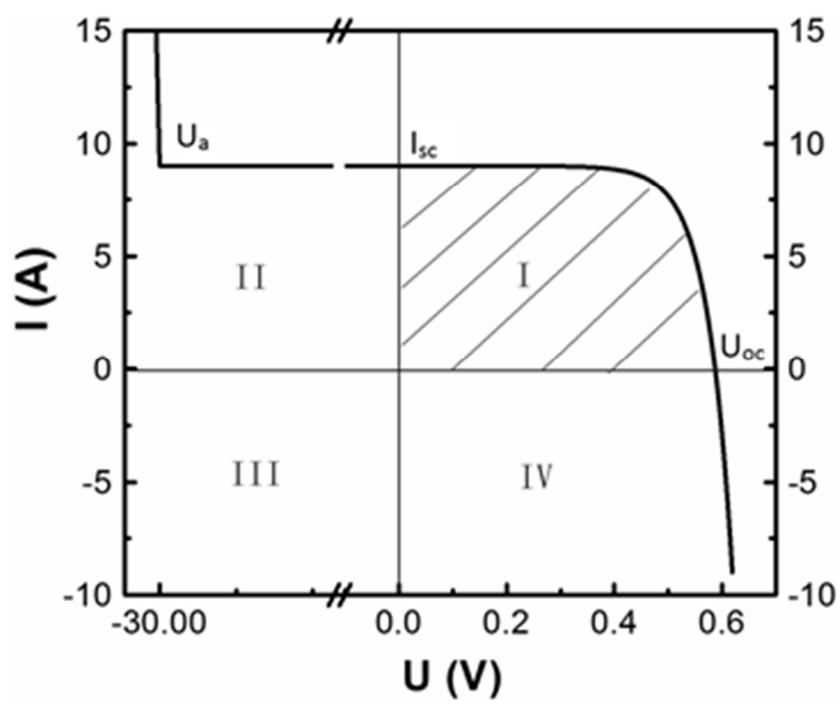

Figure 1. Schematic diagram of IV curve of photovoltaic cell in three quadrants."

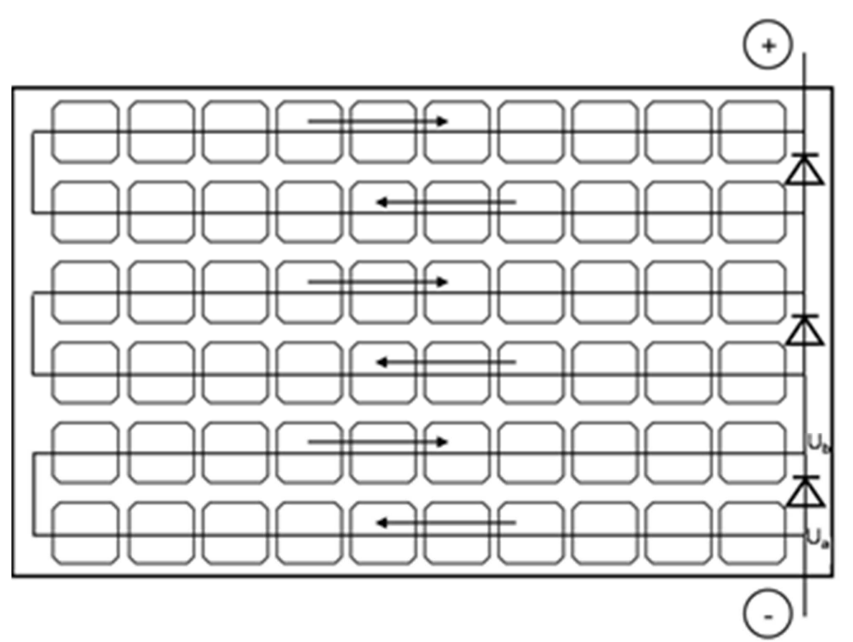

Figure 2. Common internal electrical connections of crystalline silicon photovoltaic modules.

In general, power plant crystalline silicon photovoltaic modules are connected in series by a number of single cells. Figure 2 shows the most common internal electrical 
connection structure of $250 W_{p}$ photovoltaic modules on the market at present: $10 \times 60$ photovoltaic cells in series constitute a module, the rated voltage of the module is equal to the sum of the rated voltage of each battery, and the rated current is equal to the rated current of a single battery; each 20 battery strings will be connected with a diode in parallel, when the string becomes a diode due to the damage or shielding of some batteries. When the load is consumed, the diode will short circuit the whole string. The forward threshold voltage of PV bypass diode is low (zero to several volts), the on resistance is very low, the maximum forward current is high, and the reverse breakdown voltage is high. Its working principle is as follows: when the photovoltaic cell generates electricity normally, its current direction is as shown by the arrow in Figure 2. The voltage drop direction is opposite to the current direction. At this time, the positive voltage $\left(U_{a}\right)$ of the diode is lower than the negative voltage $\left(U_{b}\right)$, and it is in the reverse cut-off state; when the photovoltaic cell loses or blocks the output current, and the load is formed, the voltage drop direction is the same as the current direction, such as: ff the positive voltage $\left(U_{a}\right)$ of the diode is higher than the negative voltage $\left(U_{b}\right)$ at least above its positive threshold voltage, the diode is on and the 20 batteries are short circuited.

Figure 3 is a schematic diagram of the most common electrical connection mode of photovoltaic power station (or sub square array): several battery components are connected in series to form a string; then several strings are connected in parallel to enter a combiner box; finally, several combiner boxes are connected in parallel to the inverter for inverter / boost. In general, the PV inverter at the power station level has the function of maximum power point tracking (MPPT), which may be MPPT for all combiner boxes together or shunt. Compared with the series photovoltaic cells, the current of each branch will be redistributed after the multi-channel parallel confluence, which leads to further deviation of each cell from its optimal working state and further reduction of the total power and efficiency of the system.

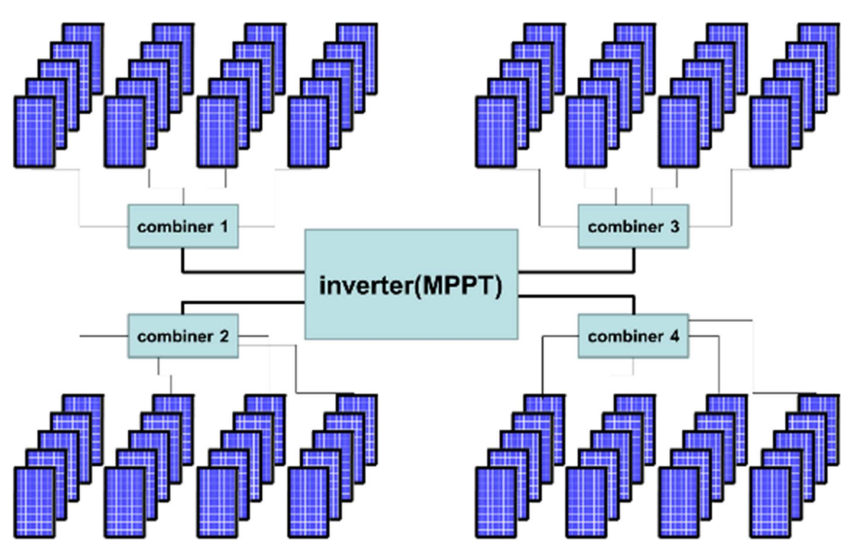

Figure 3. Common electrical topology of photovoltaic power station.

\subsection{Modelling Mathematical Methods and Program Flow}

According to the principle and internal structure of photovoltaic cell, it can be equivalent to an equivalent circuit composed of photocurrent source, diode, series resistance and parallel resistance [7], and its volt ampere characteristics can be expressed by formula (1):

$$
I=I_{L}-I_{0}\left(e^{q\left(V+I R_{S}\right) I n k T}-1\right)
$$

Where, $\mathrm{I}$ is the current, $\mathrm{V}$ is the voltage, $I_{0}$ is the saturation current of p-n junction, $I_{L}$ is the current source output current, $V+I R_{s}$ is the voltage added to both ends of $\mathrm{p}-\mathrm{n}$ junction, $\mathrm{k}$ is the Boltzmann constant $\left(1.38 \times 10^{-23} \mathrm{~J} / \mathrm{k}\right), \mathrm{T}$ is the thermodynamic temperature $(\mathrm{k}), \mathrm{q}$ is the electronic charge $(1.6$ $\times 10^{-19} \mathrm{C}$ ). It can be seen that (1) is not an explicit function, and the approximate solution obtained by Newton iteration and other methods is needed in numerical calculation [12]. Although this method can get very high precision results, but for the power plant level modelling and simulation, the amount of calculation is too large, the time-consuming and consumed computing resources are beyond the normal acceptable range. Therefore, it is necessary to deduce the display expression of volt ampere characteristics by approximation.

Using the data of short circuit point, open circuit point and maximum power point provided by the battery manufacturer, the five parameter closed model of photovoltaic cell can be built according to formula (1) [8], the IV value of short circuit point, open circuit point and maximum power point can be substituted into formula (1), and the slope of IV curve of short circuit point and open circuit point (i.e. series resistance and parallel resistance) can be substituted into the differential form of formula (1), and the five equations can be established The formula (2-7) is obtained

$$
\begin{gathered}
I=I_{S C}\left(1-C_{1}\left(e^{(V-D V) / C_{2} V_{O C}}-1\right)\right)+D I \\
C_{2}=\left(V_{m} / V_{O C}-1\right) / \ln \left(1-I_{m} / I_{S C}\right) \\
C_{1}=\left(1-I_{m} / I_{S C}\right) e^{-V_{m} / C_{2} V_{O C}} \\
D T=T-T_{\text {ref }} \\
D I=a\left(G / G_{r e f}\right) D T+\left(G / G_{r e f}-1\right) I_{S C} \\
D V=b \cdot D T-R_{S} \cdot D I
\end{gathered}
$$

Where I, V, $I_{S C}, V_{O C}, I_{m}$ and $V_{m}$ are the current, voltage, short-circuit current, open circuit voltage, maximum power point current and maximum power point voltage of the battery, $\mathrm{T}$ and $T_{\text {ref }}$ are the temperature and reference temperature (temperature under standard state), $\mathrm{G}$ and $G_{\text {ref }}$ are the light intensity and reference light intensity respectively, $a$ and $b$ are the temperature coefficient of short-circuit current and open circuit voltage of the battery, Rs is the series resistance. The parameters in formula (2-7) can be obtained from the product specifications provided by the battery manufacturer, which is very convenient to build models according to the actual situation of different power stations.

Referring to figure 1, formula (2-7) can represent the first and fourth quadrants of IV curve. For the convenience of expression, formula (2) is as follows:

$$
I=f(V)(V \geq 0)
$$


The part of the second quadrant in the IV curve can be expressed as a piecewise function:

$$
\begin{gathered}
I=I_{S C}\left(U_{a}<V<0\right) \\
\left.I=k\left(V-U_{a}\right)+I_{S C} V \leq U_{a}\right)
\end{gathered}
$$

The slope value of the curve that close to $90^{\circ}$ is determined according to the specific parameters of the battery. The formula (8-10) can be used to get the whole region expression form of the IV characteristic curve of single photovoltaic cell. In order to express conveniently, the formula (8-10) is as follows:

$$
I=F(V)
$$

Suppose that a bypass diode is responsible for protecting the strings composed of $\mathrm{nl}$ cells. When the strings work normally, the current of each cell is the same and the voltage is different, then its IV characteristics can be expressed by formula (12):

$$
V_{S}=\sum_{n_{1}} F^{-1}\left(I_{S}\right)
$$

Where $F^{-1}$ is the inverse function of the $\mathrm{F}$ function, $V_{S}$ and $I_{S}$ represents the voltage and current of the $\mathrm{n} 1$ battery string. If the string becomes a load and the absolute value of the reverse voltage is greater than the threshold voltage of the bypass diode, the diode is on and the string is short circuited, then its IV characteristics can be expressed by formula (13):

$$
V_{S}=-\left(V_{d}+I_{S} / k_{d}\right)\left(V_{s}<-V_{d}\right)
$$

Where $V_{d}$ is the threshold voltage of the diode, and $k_{d}$ is the slope of the IV curve (close to $90^{\circ}$ ) when the diode is conducting in the forward direction. For the convenience of expression, formula (12-13) is as

$$
V_{S}=G\left(I_{S}\right)
$$

Suppose that there are $\mathrm{n} 2$ bypass diodes (i.e. $\mathrm{n} 1 \times \mathrm{n} 2$ cells in total) in a module, there are $\mathrm{n} 3$ electrical modules in series in the square array, and M-channels in parallel. When $\mathrm{n} 2 \times \mathrm{n} 3$ is taken as $\mathrm{N}$, the problem of solving the output characteristics of the square array is transformed into the problem of solving the steady-state circuit of $\mathrm{N}$-series and m-parallel circuit elements with the IV characteristics shown in formula (14). This problem can be solved easily by programming on MATLAB platform.

Before the simulation calculation of photovoltaic matrix, the specific form of formula (11) should be determined first. In order to simplify the calculation load, we use the method mentioned in [9] to construct the discretization matrix of battery IV characteristics: first, the voltage is discretized into 1000 parts in the range of $[-\mathrm{Ua} \sim 2 \mathrm{Voc}]$, then $[\mathrm{T}]$ and $[\mathrm{G}]$ are brought into formula (11), and the current and voltage matrix of each cell is obtained, that is, formula (15):

$$
M V_{p, q}=\left[\begin{array}{cccc}
V_{11} & V_{12} & \ldots & V_{1 q} \\
V_{11} & V_{12} & \ldots & V_{2 q} \\
& \ldots & \ldots & \\
V_{p 1} & V_{p 2} & & V_{p q}
\end{array}\right]
$$

$$
\begin{gathered}
\left(p=n_{1} \times N \times M, q=1000\right) \\
M I(i, j)=F_{i, j}(M V(i, j))
\end{gathered}
$$

Among them, MV and MI are the current and voltage matrices of single cell, both of which are in the dimension of $\mathrm{p} \times \mathrm{q}$. Each row of elements represents the continuous change value of voltage and corresponding current value on the defined interval; each column of elements represents the total number of single cells in the photovoltaic matrix. $F_{i, j}$ is the I $\mathrm{V}$ relation function of different cell, i.e. formula (11) with different constant parameter values. When the program is running, MV and Mi exist in memory in the form of structure. When calculating the output parameters of single battery, the results can be obtained directly by linear interpolation.

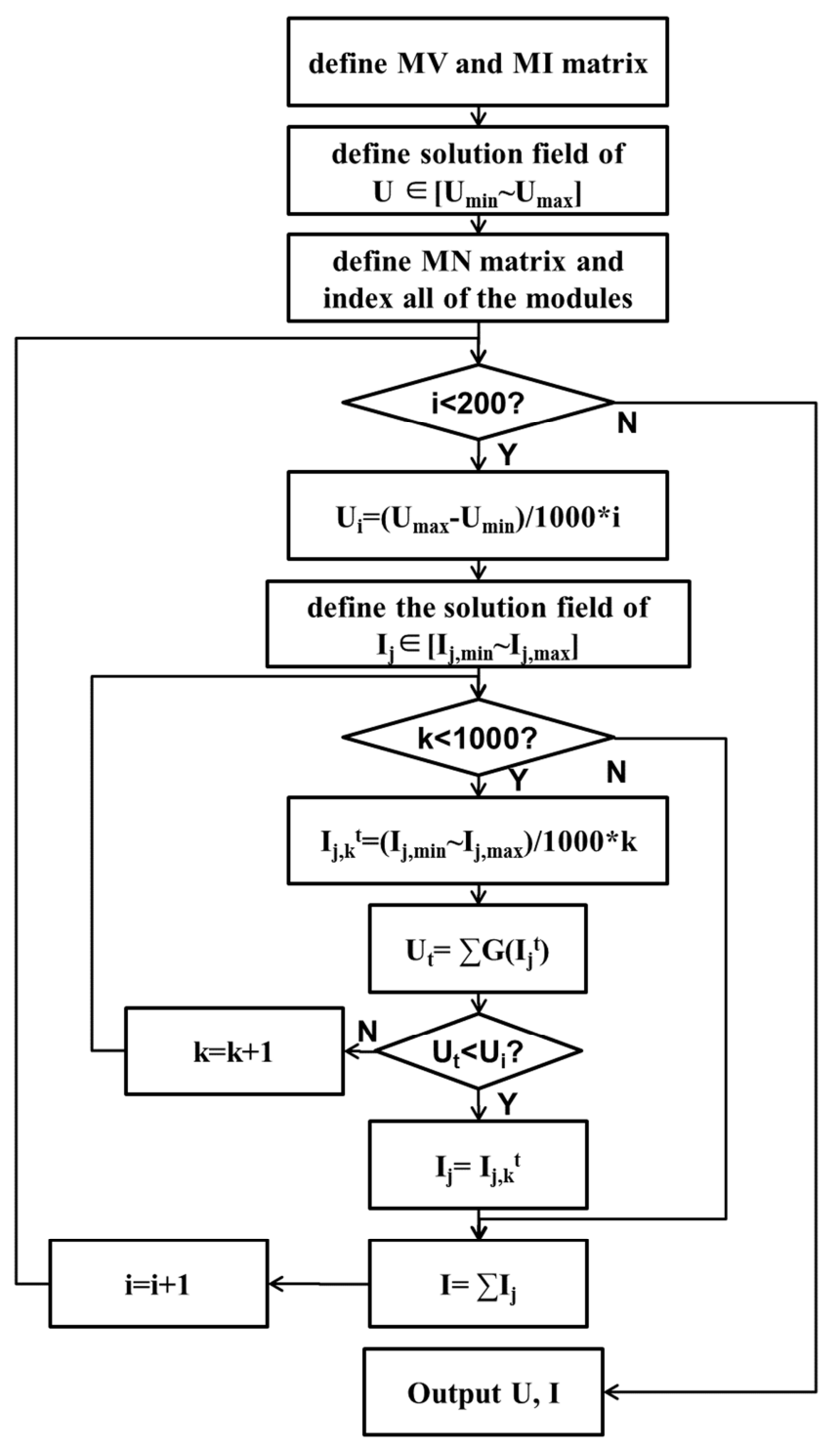

Figure 4. Flow chart of calculation program.

The flow chart of the calculation program is shown in Figure 4: after constructing the MV and MI matrix, completing parameter initialization, numbering each battery and establishing the mapping relationship with MV and MI, start to solve the output parameters of the square array (array 
$\mathrm{U}$ and I); for each voltage value $U_{i}$, solve the current of each parallel branch $I_{j}(1 \leq \mathrm{j} \leq \mathrm{N})$. The method is to discretize the solution interval and increase it from smallest to largest $I_{j}$, and substitute the test value of $I_{j}$ into formula (14) to calculate the voltage value $U_{t}$ of the whole branch (varying from the largest to smallest $I_{j}$ ). When $U_{t}$ is less than $U_{i}$, the test value of $I_{j}$ at this time is the approximate solution of parallel branch current; finally, array u and I are output as the final result.

\section{Simulation Analysis of Photovoltaic Matrix Under Partial Occlusion}

\subsection{Partial Occlusion Photovoltaic Matrix Model}

Using the above modelling method, we take a $50 \mathrm{KW}$ photovoltaic matrix as an example to analyse the influence of local occlusion on the total output power of the array. In the design of ground photovoltaic power plant, the influence of the placement of photovoltaic modules on the power generation of the system is usually considered. In recent years, the mainstream design idea is that the lateral placement of components can improve the total output power of the array in the case of early and late light occlusion more than the longitudinal placement. The reason is that in most of the photovoltaic modules produced by manufacturers, the batteries are connected in series in the longitudinal direction (as shown in Figure 2), and several series circuits are connected in parallel in the transverse direction. When the sun height angle is low in the morning and evening every day, the shadow of the front array may partially block some components of the back array near the ground. Suppose that in the case of shielding only a row of batteries, if placed horizontally, the batteries with light shielding belong to the same series circuit, so that the circuit is short circuited under the action of bypass diode, and only the generating capacity of one series circuit is lost; however, if placed longitudinally, the batteries with light shielding belong to different series circuits, these circuits may be short circuited by bypass diode, loss of power generation for the entire component.

In the photovoltaic matrix simulation calculation model in this paper, we purposely compare the differences between two different placement methods under different occlusion conditions, as shown in Figure 4. Figure 4 (a) shows the photovoltaic square array of horizontal installation of modules, with 5 columns in total. Each array contains 21 strings $\times 2$ photovoltaic modules with a rated power of $250 \mathrm{wp}$ (the internal structure of the module is consistent with figure 2 ), which are installed in 14 rows $\times 3$ rows, with a total installed capacity of $52.5 \mathrm{KW}$. In certain cases, except for the first array, there is a battery array at the bottom of each array which is blocked by the front array, and the blocked area accounts for $5.56 \%$ of the total effective battery area. Figure 4 (b) shows the photovoltaic square array with horizontal installation mode of modules. Each array consists of 20 series $\times 2$ photovoltaic modules with rated power of $250 \mathrm{wp}$, which are installed in 20 series $\times 2$ rows. The total installed capacity is $50 \mathrm{KW}$. Other conditions are the same as Figure 4 (a), and the shielding area accounts for $5 \%$ of the total effective generation battery area. In the figure, five photovoltaic matrices converge into a square array for maximum power point tracking.

In the calculation, the irradiated light intensity of the un-shaded part is set as $1000 \mathrm{~W} / \mathrm{m}^{2}$, the surface temperature of the battery is $45 \square$; the irradiated light intensity of the occluded part is set as $200 \sim 800 \mathrm{~W} / \mathrm{m}^{2}$, and the surface temperature of the battery is $35 \square$. Different environmental conditions of the power plant are considered in the setting of the value range of the light intensity of the shielding part: the total light intensity of the photovoltaic cell surface can be divided into three components: direct, scattering and reflection. According to the reference meteorological data provided by Meteonorm5.1X software, the proportion of direct and scattered light intensity in the surface radiation varies in the range of 1.7-2.5:1; the reflection intensity depends on the surrounding environment. For the ground reflection, the reflection intensity of vegetation, sand and cement ground increases in turn, which can reach about $10 \%-40 \%$ of the direct light intensity. In low latitude areas, it is also possible There is a back panel reflection of the front component. If there is a glass curtain wall around, which may exist in the photovoltaic project of the roof in the city, the reflection intensity may also be greatly enhanced. The occlusion between components can only block the direct light and part of the reflected light, and the scattering will not be blocked. Based on this, it can be estimated that the occluded light should account for 30\% 80\% of the total solar radiation intensity, usually about $40 \sim 60 \%$.

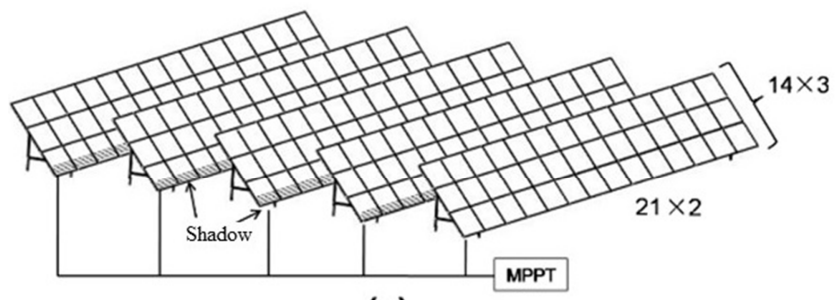

(a)

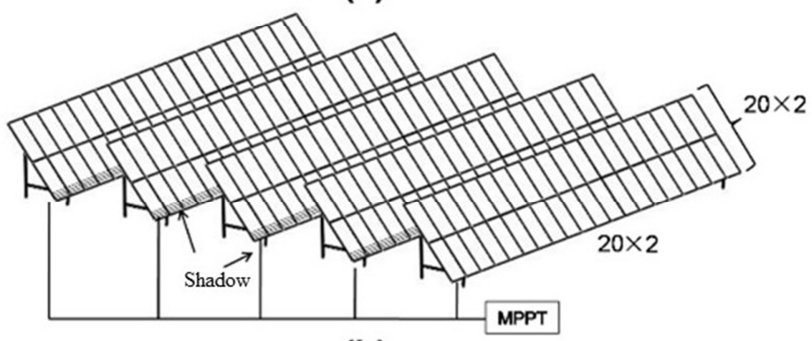

(b)

Figure 5. Schematic diagram of photovoltaic matrix modelling. (a) Horizontal installation of photovoltaic modules, (b) vertical installation of photovoltaic modules.

\subsection{Simulation Results and Analysis}

The simulation results of two different installation methods are shown in Figure 6 and Figure 7. Figure 6 (a) shows the IV curve of PV module under different light conditions when it is installed horizontally. This obvious step curve shape is similar 
to some research results about the influence of local shadow on the performance of PV module [8-11]. In the first stage, when the total voltage of the square array is low, the current of the normal battery is close to its short-circuit current, which is much higher than the short-circuit current of the blocked battery. Therefore, the blocked battery is in the reverse cut-off state (see Figure 1), and the reverse voltage of the battery string reaches the forward conduction voltage of the bypass diode, so that the string is short circuited When a load with a very low resistance has little effect on the current of the whole array series circuit, so the current of the array is basically the same as that without local shielding; 2) in the second stage, the total voltage of the array increases, but the current of the array series is still greater than the short-circuit current of the shielding battery chip, and the bypass diode continues to conduct in a positive direction, because the battery chip (not covered) in the working access state is actually in operation. The number of battery cells) is less than the total installed capacity, so the working voltage allocated to each battery cell is actually close to or more than the maximum power point on its own IV curve, that is, the inflection point of the rapid drop of current in the first quadrant of Figure 1, so the total current begins to drop rapidly; in the third stage, the total voltage of the array continues to drop, and the operation of the battery cell in this state is not blocked at this time. The current has been less than the short-circuit current of the occluded battery, the bypass diode of the occluded string is no longer on, and the occluded battery starts to output power, so the total current of the square array suddenly slows down with the increase of the voltage (step inflection point in Figure 6), and finally reaches the open circuit state.

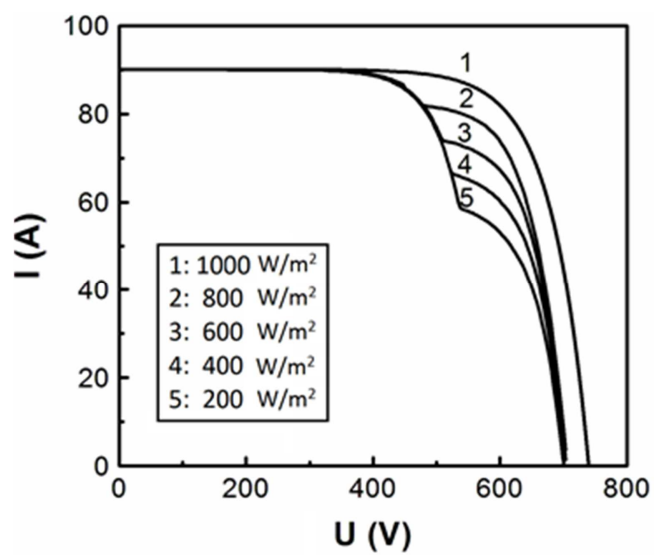

Figure 6. IV curve of horizontally installed photovoltaic matrix after partial shielding.

Figure 7 shows the IV curve of photovoltaic modules installed longitudinally under different light conditions. Unlike Figure 6, the IV curve of this model does not appear step shape, but is close to the IV curve of ordinary batteries or modules. The reason for this difference is: in the longitudinal installation mode (Figure 6 (b)), the blocked cells in the same module belong to the circuits protected by three bypass diodes. If the bypass diodes reach the forward conduction triggering condition, the three series circuits must be short circuited at the same time, that is, the whole module will no longer generate electricity; at this time, the column next to the blocked photovoltaic matrix consists of 20 modules. The series of components will become a passive load with a resistance of about $30 \Omega$. But in fact, this situation cannot happen in a photovoltaic matrix composed of multiple strings in parallel, because once the whole string becomes a pure load, other strings in parallel with it will supply power to it (the battery state enters the fourth quadrant of Figure 1), so the potential direction will be opposite to the normal working direction of the battery, and the bypass diode will return to the reverse cut-off state. For the above reasons, when the photovoltaic modules are installed longitudinally, the batteries in each series circuit are in the power generation working state regardless of whether they are blocked; after the parallel connection of each series circuit, the current is redistributed, and the power generated by each battery is lower than the power value in its separate working state; the current value of the square IV curve is significantly lower than that in all batteries from the beginning, and with the voltage The improvement of the system gradually smoothly drops to the open circuit state.

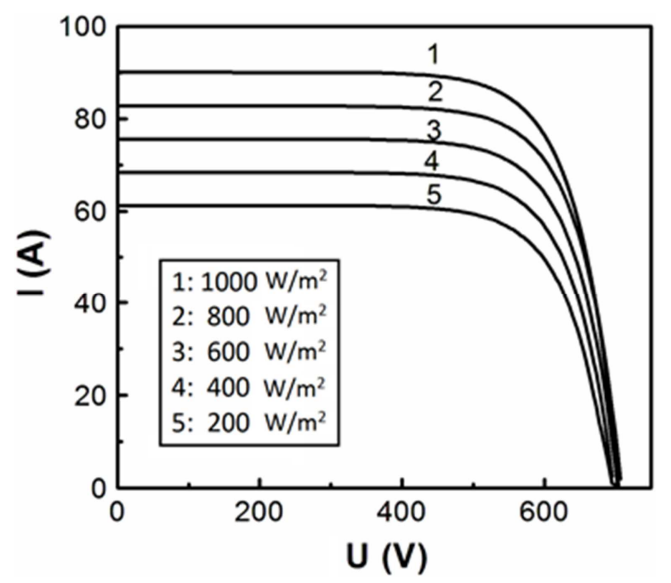

Figure 7. IV curve of longitudinally installed photovoltaic matrix after partial shielding.

Figure 8 compares the difference of the maximum power of the two installation modes under different occlusion conditions. Due to the slight difference in the total installed capacity (42 components per array are installed horizontally and 40 components are installed longitudinally), the average power of a single battery is used. When the illumination intensity of the occluded battery is greater than $600 \mathrm{~W} / \mathrm{m}^{2}$, the average power difference between the two installation methods is not significant (the power of the longitudinal installation is slightly greater than that of the transverse installation, mainly due to the shielding ratio of the longitudinal installation array is slightly higher than that of the transverse installation), which decreases approximately linearly with the decrease of the illumination intensity of the occluded area; when the illumination intensity of the occluded battery is less than $600 \mathrm{~W} / \mathrm{m}^{2}$, The average power of the transverse array decreases with the decrease of the light intensity, and tends to a fixed value (about $80 \%$ of the total 
power without occlusion), while the average power of the longitudinal array continues to decrease with the decrease of the light intensity with the same slope. When the illumination intensity of the occluded cell is $200 \mathrm{~W} / \mathrm{m}^{2}$, the total power of the horizontal array is increased by about $25 \%$ compared with that of the vertical array. The calculation results show that for the photovoltaic power plants with high atmospheric scattering rate and high ground reflectance, the influence of the two installation methods on the total power is not different; while for the photovoltaic power plants with low atmospheric scattering rate and high ground reflectance, the advantages of the horizontal installation method are obvious.

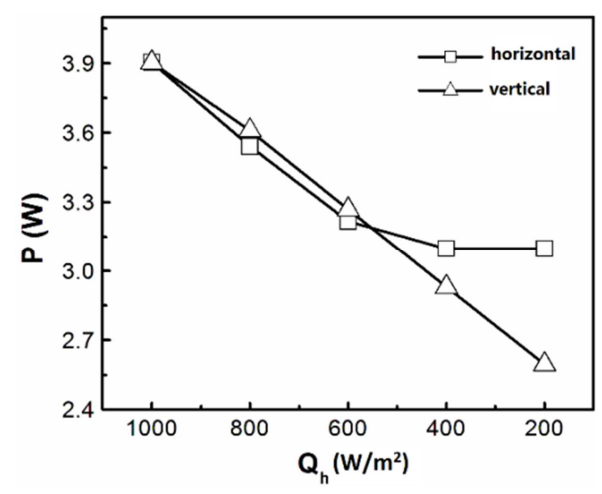

Figure 8. The relationship between the average output power of single-chip battery and the light intensity of the blocked area under different installation modes.

\section{Conclusion}

In this paper, a four-level photovoltaic power prediction modelling method including cell module string array is introduced, which is suitable for photovoltaic matrix or small power station with independent MPPT function. By numbering each cell in the square array, the model can study the influence of environmental factors (such as temperature gradient distribution, partial occlusion, etc.) that lead to the performance deviation of the cell in any geometric position on the output power of the whole square matrix. In the modelling, the expansion of IV curve of single battery in quadrant II and quadrant IV, the influence of bypass protection inside the module and current anti reverse protection between the modules are considered. This makes the calculation results simulates the actual operation of the power station quite well. Finally, using this modelling method, the difference of the total power of photovoltaic modules installed in horizontal or vertical direction under the condition of shielding between arrays is studied. The results show that when the light intensity loss in the occluded area is less than $40 \%$, there is little difference between the two installation methods; with the increase of the light intensity loss, the power loss of the transverse installation method is smaller.

\section{References}

[1] J. L. Zhou, B, Wang, Y. M. Zhang, "Parameter identification and output power prediction of photovoltaic matrix based on measured data," Renewable energy, 10 (7): 1-4, 2012.

[2] X. Y. Yang, X. H. Liu, "Large scale grid connected solar power generation model and its application," Chinese Journal of electrical engineering, 31 (11), 2011.

[3] Y. Gong, "Study on power prediction model of photovoltaic module based on parameter identification," Chengdu: University of Electronic Science and Technology of China, China, 2010.

[4] Y. T. Tan, D. S. Kirschen, N. Jenkins, "A model of PV generation suitable for stability analysis," IEEE Trans. Energy Convers, 19 (4): 748-755, 2004.

[5] M. G. Villalva, J. R. Gazoli, "Comprehensive Approach to Modeling and Simulation of Photovoltaic matrixs," IEEE Transctions on Power Electronics, 24 (5), 2009.

[6] D. G. Liu, et al. "Overview of photovoltaic power generation system model," Grid technology, 35 (8): 47-51, 2011.

[7] J. H. Su, S. J. Yu, W Zhao, et al. "Investigation on engineering analytical model of silicon solar cells," Acta energy Solaris Sinica, 20 (5): 409-412, 2005.

[8] K. Ding, Q. X. Zhai, J. W. Zhang, et al. "A model for estimating the output power of photovoltaic modules," Renewable energy, 32 (3): 275-277, 2014.

[9] R. L. Chen, Y. Cui, D. Y. Li, et al. "Study on simulation model of photovoltaic modules under uneven illumination," Journal of system simulation, 20 (7): 1681-1690, 2008.

[10] J. L. Xiao, Z. Xu, C. Lin, et al. "Optimal design of photovoltaic matrixs under partial shading," Proceedings of the CSEE, 29 (11): 119-124, 2009.

[11] X. Y. Liu, X. M. Qi. "Modeling and analysis of photovoltaic matrix under local shadow condition," Grid technology, 34 (11), 2010.

[12] H, Patel, V, Agarwal. "Matlab-based modeling to study the effects of partial shading on PV array characteristics," IEEE Trans on Energy Conversion, 23 (1): 302-310, 2008. 